\title{
The phenomenology of real and illusory tip-of-the-tongue states
}

\author{
BENNETT L. SCHWARTZ, DONALD M. TRAVIS, and ANTHONY M. CASTRO \\ Florida International University, Miami, Florida \\ and \\ STEVEN M. SMITH \\ Texas A\&M University, College Station, Texas
}

\begin{abstract}
The tip-of-the-tongue state (TOT) is the phenomenological experience that a word is on the verge of being recalled. Most research has been directed at TOT etiology and at retrieval processes occurring during a TOT. In this study, TOT phenomenology was examined. In Experiment 1, strong TOTs were more likely than weak TOTs to be followed by correct recognition, and resolution (later recall) of TOTs was higher for strong than for weak TOTs, but only for commission errors. In Experiment 2, emotional TOTs were more likely to be resolved and recognized than nonemotional TOTs. In Experiment 3, imminence was defined as the feeling that retrieval is about to occur. Imminent TOTs were more likely to be followed by resolution and recognition than were nonimminent TOTs. Illusory TOTs (TOTs for unanswerable questions) tended to be weaker, less emotional, and less imminent than TOTs for answerable questions.
\end{abstract}

Most people report experiencing a tip-of-the-tongue state (TOT) on a common basis (A. S. Brown, 1991; Reason \& Lucas, 1984). To the rememberer, a TOT is a strong feeling that a particular target word can be retrieved, sooner or later. Researchers have used the term TOT in two senses. The first is that a TOT is the feeling that we know a particular target word and the feeling that the retrieval of that target is imminent. This is the sense in which we use the term in this paper. On the other hand, some research has defined TOTs as the state of having partial knowledge about an item for which we are experiencing a feeling of knowing (see Schwartz, 1999; Vigliocco, Antonini, \& Garrett, 1997). In general, these two approaches are compatible, because all studies have shown that TOTs are correlated with objective indices of memory, such as retrieval of the first letter, a syllable, grammatical gender, synonyms, homonyms, or semantically related information (Brennan, Baguley, Bright, \& Bruce, 1990; A. S. Brown, 1991; Koriat \& Lieblich, 1974; Miozzo \& Caramazza, 1997; Vigliocco et al., 1997). When rememberers experience TOTs, they are more likely to (1) retrieve partial information, (2) recognize the correct target, and (3) eventually recall the target (known as TOT resolution) than when the rememberer is

The authors thank Michelle Vigoa and Daniel Yero for their assistance in collecting and analyzing data, Juan Gonzalez and the participants of the 12th annual Florida Cognition Conference for helpful discussion, and Leslie Frazier, Asher Koriat, Ravit Levy-Sadot, and two anonymous reviewers for helpful comments on earlier drafts of this paper. Correspondence concerning this article should be addressed to B. L. Schwartz, Department of Psychology, Florida International University, Miami, FL 33199 (e-mail: schwartb@fiu.edu). not experiencing a TOT (see A. S. Brown, 1991; Smith, 1994, for reviews).

Research has used the retrieval of target information to divide TOTs into substates. For example, Koriat and Lieblich (1974) divided TOTs into nine different substates, depending on whether the target was recognized or not recognized, whether it was recalled or not recalled, and whether partial information was recalled or not recalled. Jones and Langford (1987) divided TOTs into objective TOTs (those for which target information was retrieved) or subjective TOTs (those for which no target information was retrieved; see, also, Perfect \& Hanley, 1992). Burke, MacKay, Worthley, and Wade (1991) defined "proper" TOTs as those followed by successful recognition. Vigliocco et al. (1997) divided TOTs into positive or negative TOTs, depending on whether the participant could correctly verify the TOT target. To the rememberer, however, it is the intense feeling that marks the TOT. Because the TOT is a subjective state and not simply a marker of partial accessibility, the phenomenology of TOTs, separate from the phenomenology of retrieval, is important. Indeed, it is likely that the TOT feeling is not uniform across all instances and occasions. It may vary with respect to intensity, emotional content, and feeling of imminence. The present study addresses whether people can distinguish between phenomenological substates of the TOT and whether these substates are predictive of memory performance.

Researchers have noted the importance of tip-of-thetongue (TOT) phenomenology but have been content to examine it anecdotally. Informally, the TOT feeling has been described by numerous researchers, dating back to William James, who wrote that the TOT makes us "tingle 
with our sense of closeness" (James, 1890, p. 251). R. Brown and McNeill (1966) described TOTs in the following way: "The signs of it [TOT] were unmistakable; he would appear to be in mild torment, something like the brink of a sneeze, and if he found the word his relief was considerable" (p. 326). A. S. Brown (1991) wrote that "such descriptions [of the TOT] are congruent with one's personal introspections of inner turmoil when grappling for an elusive word" (p. 205). However, no empirical research has been directed at whether tingling, torment, or turmoil is involved in the experiencing of TOTs. We will begin to address issues of phenomenology in this paper. Before we introduce the particular aspects of the TOT that we studied, we will describe why the emphasis has not been placed on TOT phenomenology in most TOT research.

The neglect of TOT phenomenology has occurred for several reasons. First, many researchers make use of TOTs as a way of studying retrieval processes. TOTs are interesting because they serve as "windows" on the process of retrieval (Kohn, Wingfield, Menn, Goodglass, Berko Gleason, \& Hyde, 1987) or because they offer "slowmotion photography" of the retrieval process (A. S. Brown, 1991). In this research, the phenomenology of TOTs is secondary to the interest in word retrieval, Second, other researchers are interested in the etiology of the TOTs. Experimental variables are manipulated, and the frequency of TOTs is observed (e.g., Harley \& Bown, 1998; Schwartz \& Smith, 1997). The phenomenology is secondary to the cognitive processes that cause the TOT. Both of these research agendas may neglect the phenomenology of TOTs because of an implicit belief in what Tulving (1989) called the doctrine of concordance (see Schwartz, 1999). According to Tulving, research typically assumes a correlation between cognition and phenomenology. By understanding the processes of cognition, one explains the phenomenology. There is now research, however, that suggests that TOTs and retrieval processes are dissociable (Schwartz, 1999). For this reason, we think it is important to explore the phenomenology of TOTs and to examine the correlations of the phenomenology with objective performance.

One important aspect of TOT phenomenology is the intensity of the TOT feeling. Anecdotally, some TOTs are so strong that they may bother us for days. Others pass without a second thought. However, TOT research has almost exclusively used dichotomous judgments, either yes for a TOT or no for an n-TOT (the state of not being in a TOT). An exception is a study by Kozlowski (1977), who found that "extreme" TOTs were followed by higher recognition of unrecalled targets than were "moderate" TOTs or n-TOTs (see, also, Gardiner, Craik, \& Bleasdale, 1973). Thus, it seems likely that rememberers can and do distinguish intensities of TOTs, similar to the distinctions they can make between stronger and weaker feelings of knowing. In the present study (Experiment 1), participants were asked to distinguish between strong and weak TOTs. We also measured several other objective and subjective measures of memory, to see whether intensity of a TOT would predict other characteristics of memory and metamemory.

At this point, it is important to reiterate the differences between TOTs and feelings of knowing (see A. S. Brown, 1991). Feeling-of-knowing judgments are predictions of recognition (or sometimes recall) and can be given for any unrecalled item. TOTs, by contrast, are predictions of recall and occur involuntarily on the part of the rememberer (A. S. Brown, 1991). In the present study, we will elicit both TOTs and feeling-of-knowing judgments. Typically, TOTs are thought of as occurring spontaneously in a rememberer when a known item is not retrieved, whereas feeling-of-knowing judgments can occur for any item and are specifically called for by the experimenter.

A second important phenomenological aspect of TOTs is the extent to which TOTs evoke an emotional response. Many researchers and textbook writers have commented on the emotional sense of frustration that sometimes accompanies a TOT. Anecdotally, people report that TOTs are frequently accompanied by an emotional feeling. However, A. S. Brown (1991) wrote that "this emotional arousal may simply reflect a reaction to the momentary retrieval failure rather than being an integral component of the TOT"' (p. 205). Nonetheless, we know of no research that has specifically looked at the relation between TOTs and emotion. The present study will attempt to redress this oversight. In Experiment 2, the participants were asked to mark those TOTs that were accompanied by feelings of emotion and those that were not. In order to substantiate emotions during TOTs, we then tested whether emotionality in TOTs would predict other measures of memory and metamemory.

A third important phenomenological aspect of TOTs is the importance of a sense of imminence to recall. R. Brown and McNeill (1966) defined the TOT as the feeling of being on the verge of recall. Smith (1994) described this feeling of being on the verge of recall as a feeling of imminence. However, he argued that not all TOTs may be imminent ones. Anecdotally, we have all experienced TOTs, been unable to recall the target word immediately, only to have it "pop" into consciousness some time later (Burke et al., 1991). In Experiment 3, we asked rememberers to distinguish between imminent and nonimminent TOTs. We also assessed other measures of memory and metamemory, to see whether the sense of imminence is predictive.

\section{REAL AND ILLUSORY TIP-OF-THE-TONGUE STATES}

Typically, TOT research has assumed that TOTs reflect the activation of an unrecalled but represented target word (Burke et al., 1991; Harley \& Bown, 1998; Kohn et al., 1987). Recent research, however, illustrates the potential for dissociations between the process of re- 
trieval and the experience of a TOT (Metcalfe, Schwartz, \& Joaquim, 1993; Schwartz, 1998; Schwartz \& Smith, 1997; Widner, Smith, \& Graziano, 1996). Schwartz (1998) demonstrated the existence of illusory TOTs, which are phenomenological experiences in which the person feels that the target is memorable, but the target memory is either unavailable, forgotten, or was never learned (see, also, Smith, 1994). In Schwartz' (1998) study, unanswerable questions (What is the name of the planet Mercury's moon), embedded among normal general information questions, induced illusory TOTs on approximately $15 \%$ of the questions. Schwartz (1998) argued that the existence of illusory TOTs provides support for the dissociability of TOTs and the process of retrieval.

In the present study, we wish to identify whether the phenomenology of illusory TOTs is separable from the phenomenology of TOTs for answerable questions. In the three experiments here, unanswerable questions have been embedded among the normal general information questions. We suspect that the etiology of illusory TOTs may be based more on inferential strategies than are other TOTs. Therefore, illusory TOTs may be less intense, less emotional, and less imminent than TOTs to answerable questions, because the latter may also be driven by activation of a target or by partial retrieval of target attributes.

The goal of the present study was to explore TOT phenomenology by asking rememberers to make distinctions concerning TOT substates. In the first experiment, rememberers distinguished between stronger and weaker TOTs. We predicted that the more intense TOTs would be associated with a greater likelihood of resolution and recognition and with a higher feeling of knowing. In the second experiment, we predicted that more emotional TOTs would be associated with better recognition, but not necessarily with better resolution. We suspected that emotion in TOTs may be associated with retrieval blocking, which will lower the likelihood of resolving the emotional TOT but should not interfere with recognition of the target. In the third experiment, we predicted that imminent TOTs would be associated with better resolution and better recognition than were nonimminent TOTs. We examined the phenomenological characteristics of illusory TOTs in each experiment. We expected it to be more likely that illusory TOTs would be assigned to the weak, less emotional, and less imminent TOT category.

\section{EXPERIMENT 1}

The purpose of Experiment 1 was to assess whether participants distinguish between strong and weak TOT substates, which our pilot work suggested was a sensible division of TOTs. In this experiment and in the ones that follow, initial TOT judgments were followed by a second chance to recall the target (which gave us a resolution measure) and by an opportunity to recognize the target from among alternatives. In this experiment, we predicted that strong TOTs would be associated with both better resolution and recognition of the target word.

\section{Method}

Participants. The participants were 41 Florida International University students, who received partial course credit for their participation. Each participant was tested individually on a Macintosh computer during a session that lasted approximately $1 \mathrm{~h}$.

Materials. The stimuli for the experiment were 80 general information questions taken from the Nelson-Narens norms (Nelson $\&$ Narens, 1980). These questions will be referred to as the answerable questions. The 20 unanswerable questions were selected from earlier work (see Schwartz, 1998). The unanswerable questions were constructed to sound plausible but did not have any possible answer. They were also constructed to appear similar to the questions from the Nelson-Narens norms. Thus, one answerable question was, "For which country is the rupee the monetary unit?" The corresponding unanswerable question was, "For which country is the jaque the monetary unit?" Previous testing indicated that the answerable 80 questions were neither too easy nor too difficult for the student population (Schwartz, 1998). The list of items was randomized for each participant. Each participant responded to each of the 100 questions ( 80 answerable plus 20 unanswerable).

Procedure. The participants were first given detailed instructions about the procedure. They were told that they would be answering a series of general information questions, some of which would be easy and some of which would be more difficult. They were given an explanation of what the term tip of the tongue meant. All the participants reported being familiar with the experience and with the term. The instructions were as follows:

If you do not answer the question correctly or leave the answer blank, you will be asked whether or not you are in a tip-of-the-tongue state for the target answer. A tip-of-the-tongue state (abbreviated TOT) means that you feel as if it is possible that you could recall the target answer, and that you feel as if its recall is imminent. Sometimes the tip-of-thetongue state may feel particularly strong; other times, the tip-of-tongue state may not feel as strong. If you feel as if you are in a strong TOT state, please press the $S$ key after the prompt that asks you if you are experiencing a TOT. If you feel as if you are in a weak TOT state, please press the $\mathrm{W}$ key. If you are not in a TOT state, please press the $\mathrm{N}$ key

This was explained first by the experimenter and then was repeated on the computer just before the experiment began. The experimenter then started the computer program that ran the experiment.

Each question appeared on the middle of the screen, and a prompt appeared beneath the question. The question remained on the screen until the participants typed in their responses. The participants typed in their responses, or they indicated that they did not know by typing in a question mark. If the participants typed in the correct response, they simply moved on to the next question. If they indicated that they did not know (omission error) or answered incorrectly (commission error), they were asked whether or not they were in a TOT. The participants typed in " $S$ " when they were in a strong TOT, a " $W$ " when they were in a weak TOT, and an " $N$ " when they were not experiencing a TOT.

After the participants attempted retrieval for all 100 questions and made TOT judgments for those they did not recall correctly, they were given more instructions. They were told that they would be asked again whether they could answer the general information questions. If they could not recall the answer, they would again be queried about whether or not they were in a TOT. They were also asked to make a feeling-of-knowing judgment for that item. A feeling-of-knowing judgment was defined as a prediction of successful recognition (see Nelson, 1988). The participants were told that they would see the correct answer to the question among a total 
of eight answers, and they were to judge on a scale of 0 to 100 whether they would be able to select the correct answer. This second recall/ TOT/feeling-of-knowing judgment phase included both the answerable and the unanswerable questions.

Finally, after completing the recall/TOT/feeling-of-knowing judgment phase, the participants were given a final recognition test for the answerable questions, but not for the unanswerable ones. They were again shown the question, followed by eight alternatives, one of which was the correct answer (Wilkinson \& Nelson, 1984). Each alternative was accompanied by a number. The participants typed in the number associated with the answer that they thought was correct. They were then presented with the next question. This continued for all the originally missed questions, minus those that had been successfully resolved during the second recall phase. At the end of the session, the participants were thanked for their participation, fully debriefed, and given credit in their introductory psychology course.

\section{Results}

Statistical reliability was measured at $p \leq .05$ in all three experiments, unless otherwise noted. We used analyses of variance (ANOVAs) throughout, even though our main variables were not true independent variables. Use of an ANOVA allowed us to examine interactions; however, we did not infer causality from any of these analyses.

Recall and recognition. Correct recall at the first test was $36 \%$. Correct recall was computed with a weak criterion for spelling. The computer program was set up to accept many misspellings. However, it could not catch all misspellings (e.g., camalion for chameleon, Skaspeater for Shakespeare). These odd misspellings were removed postexperimentally by hand.

We tracked response time in this initial recall phase as a function of whether the response was correct, a commission error, or an omission error. There was an overall main effect of response type $\left[F(2,68)=43.37, M S_{\mathrm{e}}=\right.$ 3.19]. Post hoc tests indicated that this effect was due to long response times for commission errors $(12.8 \mathrm{sec})$, relative to correct responses $(9.3 \mathrm{sec})$ and omission error responses (i.e., don't know responses; $9.4 \mathrm{sec}$ ). The mean number of commission errors for answerable questions was 26.5 per participant, whereas the mean number of omission errors for answerable question was 24.7 per participant (see Krinsky \& Nelson, 1985, for a similar analysis). Overall, recognition of initially unrecalled items was $36 \%$.

Distribution of tip-of-the-tongue states across categories. The participants readily made the distinction between strong and weak TOTs. Informal questioning indicated that few, if any, participants found the distinction unnatural or difficult to make. Some interesting patterns, however, arose in the distribution of TOTs across response category (see Table 1A). For the TOT judgment at Time 1, the participants used the strong category more than the weak category for answerable questions $[F(1,40)=33.8$, $\left.M S_{\mathrm{e}}=0.42\right]$. However, for unanswerable (illusory) questions, the effect was reversed; weak TOTs were more common than strong TOTs $\left[F(1,40)=11.45, M S_{\mathrm{e}}=\right.$ 0.14]. TOTs were more common after commission errors than after omission errors $\left[F(1,34)=18.46, M S_{\mathrm{e}}=\right.$
Table 1A

Percentage of Tip-of-the-Tongue States (TOTs) for Answerable Questions and Unanswerable Questions at Judgment 1 and Judgment 2 for Experiment 1

\begin{tabular}{lccccccc}
\hline & \multicolumn{2}{c}{ Answerable Questions } & \multicolumn{3}{c}{ Unanswerable Questions } \\
\cline { 2 - 3 } \cline { 6 - 7 } \cline { 5 - 7 } & Strong & Weak & N-TOT & & Strong & Weak & N-TOT \\
\hline Judgment 1 & & & & & & & \\
$\quad$ Combined & .34 & .20 & .46 & .07 & .15 & .78 \\
Omission & .21 & .15 & .63 & & & \\
$\quad$ Commission & .47 & .20 & .32 & & & \\
Judgment 2 & & & & & & \\
$\quad$ Combined & .32 & .27 & .41 & .09 & .07 & .84 \\
Omission & .28 & .26 & .46 & & & \\
Commission & .45 & .27 & .27 & & & \\
\hline
\end{tabular}

Note-N-TOT, no tip-of-the-tongue feeling.

Table 1B

Percentage of Tip-of-the-Tongue States (TOTs) for Answerable Questions and Unanswerable Questions at Judgment 1 and Judgment 2 for Experiment 2

\begin{tabular}{lccccccc} 
& \multicolumn{2}{c}{ Answerable Questions } & \multicolumn{3}{c}{ Unanswerable Questions } \\
\cline { 2 - 3 } \cline { 6 - 7 } & Emot & N-Em & N-TOT & & Emot & N-Em & N-TOT \\
\hline Judgment 1 & & & & & & & \\
$\quad$ Combined & .26 & .21 & .53 & .07 & .11 & .82 \\
Omission & .19 & .18 & .62 & & & \\
$\quad$ Commission & .35 & .23 & .42 & & & \\
Judgment 2 & & & & & & \\
$\quad$ Combined & .23 & .23 & .54 & .07 & .07 & .86 \\
Omission & .20 & .25 & .55 & & & \\
Commission & .34 & .22 & .43 & & & \\
\hline
\end{tabular}

Note-Emot, emotion; N-Em, nonemotional; N-TOT, no tip-of-thetongue feeling.

Table 1C

Percentage of Tip-of-the-Tongue States (TOTs) for Answerable Questions and Unanswerable Questions at Judgment 1 and Judgment 2 for Experiment 3

\begin{tabular}{|c|c|c|c|c|c|c|}
\hline & \multicolumn{3}{|c|}{ Answerable Questions } & \multicolumn{3}{|c|}{ Unanswerable Questions } \\
\hline & $\overline{I m m i n}$ & N-Im & N-TOT & Immin & $\mathrm{N}-\mathrm{Im}$ & N-TOT \\
\hline \multicolumn{7}{|l|}{ Judgment 1} \\
\hline Combined & .26 & .25 & .49 & .06 & .13 & .81 \\
\hline Omission & .15 & .22 & .63 & & & \\
\hline Commission & .41 & .32 & .27 & & & \\
\hline \multicolumn{7}{|l|}{ Judgment 2} \\
\hline Combined & .22 & .31 & .47 & .06 & .07 & .87 \\
\hline Omission & .18 & .27 & .55 & & & \\
\hline Commission & .31 & .34 & .34 & & & \\
\hline
\end{tabular}

Note-Immin, imminent; N-Im, nonimminent; N-TOT, no tip-of-thetongue feeling.

$0.19]$. For the TOT judgments at Time 2 , strong TOTs were more common than weak TOTs $\left[F(1,40)=5.14, M S_{\mathrm{e}}=\right.$ $0.10]$. For the TOTs at Time 2, TOTs after errors of commission were also more common than TOTs after errors of omission $\left[F(1,32)=35.21, M S_{\mathrm{e}}=0.10\right]$. One caveat to these findings is the high rate of TOTs (see A. S. Brown, 1991). If strong and weak TOTs are combined, the TOT rate was over $50 \%$ for answerable questions. Although this high rate suggests strong demand characteristics toward reporting a TOT, it should be noted that the general information questions used in this experiment were selected for their success at inducing TOTs. 
For the first TOT judgment, for the answerable questions, the participants experienced an average of $54 \%$ of unrecalled targets. For the unanswerable questions, the TOT rate was lower at $22 \%$. The participants were statistically more likely to experience a TOT for the answerable questions than for the unanswerable questions at both the first judgment $\left[F(1,40)=83.3, M S_{\mathrm{e}}=1.03\right]$ and the second judgment $\left[F(1,40)=68.85, M S_{\mathrm{e}}=1.89\right]$.

We did an additional analysis comparing TOT rates for unanswerable questions with TOT rates for negative TOTs (Vigliocco et al., 1997). This analysis was done only on omission errors, because commission errors were more common with answerable questions. Negative TOTs are those followed by a subsequent failure of recognition. There were significantly more negative TOTs than illusory TOTs $\left[F(1,34)=12.79, M S_{\mathrm{e}}=0.01\right]$. Moreover, more of the negative TOTs were given strong judgments $\left[F(1,34)=17.37, M S_{\mathrm{e}}=0.01\right]$.

Accuracy of tip-of-the-tongue states for answerable questions. TOTs are predictions of recall. Therefore, the purest way of measuring the accuracy of TOTs is to compare resolution rates of TOTs and n-TOTs. Resolution refers to the likelihood that a TOT was followed by subsequent recall of the target word. Resolution was measured by looking at the likelihood of recalling the target on the second recall test. Resolution was more likely to occur after a commission error $(.15)$ than after an omission error $\left[.04 ; F(1,23)=26.57, M S_{\mathrm{e}}=0.02\right]$. Moreover, when resolution was examined as a function of initial TOT substate, there was an interaction between intensity of the TOT and the likelihood of a resolution [see Ta-

Table 2 A

Likelihood of Resolving (Reminiscence) on Second-Chance Recall as a Function of Initial Error Status and Strength of Tip-of-the Tongue (TOT) Feeling for Experiment 1

\begin{tabular}{lccc}
\hline & Strong TOTs & Weak TOTs & N-TOTs \\
\hline Commission errors & .23 & .08 & .15 \\
Omission errors & .03 & .07 & .03 \\
\hline
\end{tabular}

Note-N-TOT, no tip-of-the-tongue feeling.

Table 2B

Likelihood of Resolving (Reminiscence) on Second-Chance Recall as a Function of Initial Error Status and Strength of Tip-of-the-Tongue (TOT) Feeling for Experiment 2 Emotional TOTs Nonemotional TOTs N-TOTs

\begin{tabular}{lccc} 
& & & \\
\hline Commission errors & .20 & .15 & .16 \\
Omission errors & .07 & .03 & .02 \\
\hline
\end{tabular}

Note-N-TOT, no tip-of-the-tongue feeling.

Table 2C

Likelihood of Resolving (Reminiscence) on Second-Chance Recall as a Function of Initial Error Status and Strength of Tip-of-the-Tongue (TOT) Feeling for Experiment 3 Imminent TOTs Nonimminent TOTs N-TOTs

\begin{tabular}{llll}
\hline Commission errors & .28 & .12 & .16 \\
Omission errors & .14 & .03 & .03 \\
\hline
\end{tabular}

Note-N-TOT, no tip-of-the-tongue feeling. ble $\left.2 \mathrm{~A} ; F(2,46)=6.64, M S_{\mathrm{e}}=0.02\right]$. For omission errors, there was no difference in resolution between strong TOTs, weak TOTs, and n-TOTs. However, for commission errors, strong TOTs were more likely to be followed by a successful resolution.

We also examined false resolutions; that is, we looked at the rate at which TOTs were resolved, but with the wrong target word (see Koriat, 1993). Overall, false resolution rates were low. However, false resolutions were more likely to occur after initial commission errors (.10) than after omission errors $\left[.04 ; F(1,33)=62.10, M S_{\mathrm{e}}=\right.$ $0.004]$. False resolutions were also more likely to occur after strong TOTs (.11) than after weak TOTs [.03; $\left.F(2,66)=9.65, M S_{\mathrm{e}}=0.01\right]$.

The final recognition test can also be used as a measure of accuracy. If TOTs are accurate predictors of memory storage, recognition should be higher after a TOT than after an n-TOT. Using the first TOT as the criterion TOT, recognition was higher after a strong TOT than after a weak TOT, which was higher than that after an n-TOT $\left[F(2,76)=39.89, M S_{\mathrm{e}}=0.02\right]$. Using the second TOT as the criterion TOT, recognition was higher after a strong TOT than after a weak TOT, which was higher than that after an an n-TOT $\left[F(2,76)=38.51, M S_{\mathrm{e}}=.03\right.$; see Table $3 \mathrm{~A}$ ]. A parallel method of examining accuracy of the TOTs is to look at the gamma correlation between the TOTs and the likelihood of subsequent recognition (see Nelson, 1984, for a justification of the gamma correlation). The first TOT judgments showed a .60 gamma correlation with recognition; the second TOT judgments showed a .65 correlation with recognition. The difference in accuracy between the first TOT and the second TOT was not significant $(F<1)$, but both gamma correlations were significantly higher than chance.

Feeling-of-knowing judgments. The mean feelingof-knowing judgment was $48 \%$ for the answerable questions and $24 \%$ for the unanswerable questions $[F(1,40)=$ $\left.131, M S_{\mathrm{e}}=95\right]$. The gamma correlation between the feeling-of-knowing judgments and recognition was .39 , typical for feeling-of-knowing judgments in this paradigm (see Nelson, 1988). This correlation was significantly different from zero. Feeling-of-knowing judgments were also significantly correlated with TOTs at Judgment 1 $($ gamma $=.51)$ and at Judgment $2($ gamma $=.34)$.

\section{Summary and Discussion}

The data described above yielded patterns relating the phenomenology of TOTs to other measures, both subjective and objective, of memory. First, the participants used both categories of TOTs, subjectively dividing TOTs into those that felt strong to them and those that felt weaker. TOTs to answerable questions led to approximately equal use of the strong and the weak categories, whereas unanswerable questions produced illusory TOTs that were generally rated as weak, although there were some illusory TOTs that were strong. Second, the participants' distinction between strong and weak TOTs was predictive of objective performance. Stronger TOTs were 
Table 3A

Likelihood of Recognition as a Function of Type of Tip-of-the-Tongue (TOT) Response for Experiment 1

\begin{tabular}{lccc} 
& Strong TOTs & Weak TOTs & N-TOTs \\
\hline TOT at Time 1 & .35 & .30 & .10 \\
TOT at Time 2 & .41 & .26 & .09 \\
\hline
\end{tabular}

Note-N-TOT, no tip-of-the-tongue feeling.

Table 3B

Likelihood of Recognition as a Function of Type of Tip-of-the-Tongue (TOT) Response for Experiment 2

\begin{tabular}{lccc}
\hline & Emotional TOTs & Nonemotional TOTs & N-TOTs \\
\hline TOT at Time 1 & .39 & .32 & .10 \\
TOT at Time 2 & .46 & .35 & .09 \\
\hline
\end{tabular}

Note-N-TOT, no tip-of-the-tongue feeling.

Table 3C

Likelihood of Recognition as a Function of Type of Tip-of-the-Tongue (TOT) Response for Experiment 3 Imminent TOTs Nonimminent TOTs N-TOTs

\begin{tabular}{llll}
\hline TOT at Time 1 & .33 & .31 & .10 \\
TOT at Time 2 & .50 & .30 & .08 \\
\hline
\end{tabular}

Note-N-TOT, no tip-of-the-tongue feeling.

more likely to be resolved at the time of second recall, at least for those following commission errors, and stronger TOTs were followed by better recognition. Even when the target was not the correct target, more attempted resolutions occurred after strong than after weak TOTs. These data suggest that a distinction between a strong and a weak TOT has both psychological reality and is predictive of performance.

\section{EXPERIMENT 2}

In Experiment 2, we asked the participants to make a distinction between those TOTs that were accompanied by a feeling of emotion from those that were not. We then proceeded to collect data on a number of subjective and objective memory measures, to investigate whether the distinction the participants make between different kinds of TOTs was predictive of objective retrieval performance. We also included unanswerable questions, to address whether illusory TOTs are more likely to be emotional or nonemotional.

\section{Method}

Participants. The participants were 60 Florida International University students, who received partial course credit for their participation. Each participant was tested individually on a Macintosh computer during a session that lasted approximately $1 \mathrm{~h}$. One participant's data were not included because she became ill during the testing.

Materials. The stimuli for the experiment were identical to those used in Experiment 1.

Procedure. The procedures were identical to those used in Experiment 1 , with the following exceptions. The instructions for what constituted an emotional and a nonemotional TOT were as follows:
If you do not answer the question correctly or leave the answer blank, you will be asked whether or not you are in a tip-of-the-tongue state for the target answer. A tip-of-the-tongue state (abbreviated TOT) means that you feel as if it is possible that you could recall the target answer, and that you feel as if its recall is imminent. Sometimes, you may feel frustrated or emotional that you cannot recall a word that you are sure you know. If you are in a TOT state that is accompanied by this emotional content, indicate a TOT with emotion by pressing the $\mathrm{E}$ key. If you are in a TOT state, but do not feel an emotional state, press the P key. If you cannot recall the answer and are not in a TOT state, press the $\mathrm{N}$ key.

This was explained first by the experimenter and then was repeated on the computer just before the experiment began. The experimenter then started the computer program that ran the experiment.

\section{Results}

Recall and recognition. Correct recall at the first test was 34\%, close to the mean obtained in Experiment 1. Correct recall was again computed with a weak criterion for spelling.

Response times differed as a function of whether the initial response was correct, a commission error, or an omission error $\left[F(2,104)=15.38, M S_{\mathrm{e}}=10.22\right]$. Post hoc tests indicated that this effect was due to long response times for commission errors $(13.2 \mathrm{sec})$, relative to correct responses $(9.8 \mathrm{sec})$ and omission error responses $(11.4 \mathrm{sec})$. The mean number of commission errors was 26.0 per participant, whereas the mean number of omission errors was 26.8 per participant. Overall, recognition of initially unrecalled items was $36 \%$, identical to that observed in Experiment 1.

Distribution of tip-of-the-tongue states across categories. The participants made the distinction between emotional and nonemotional TOTs. Unlike the difference between strong and weak states, several participants were confused. When they expressed confusion, the distinction between a TOT with emotion and one without emotion was further discussed. For the TOT judgment at Time 1, the participants used the emotional category slightly more often than they used the nonemotional TOTs [see Table 1B; $F(1,58)=3.53, M S_{\mathrm{e}}=0.02, p=.06$ ]. There were more TOTs at Time 1 following commission errors than there were for omission errors $[F(1,58)=$ $\left.32.36, M S_{\mathrm{e}}=0.02\right]$. However, for unanswerable (illusory) questions, the effect was reversed; nonemotional TOTs were slightly more common than emotional TOTs $\left[F(1,58)=3.54, M S_{\mathrm{e}}=0.02, p=.06\right]$. At Time 2, however, there were no differences in rates between emotional and nonemotional TOTs, but commissions still outnumbered omissions $\left[F(1,50)=31.99, M S_{\mathrm{e}}=0.02\right]$. The interaction was significant, with emotional TOTs being more likely to follow commission errors and nonemotional TOTs being more likely to follow omission errors $\left[F(1,50)=14.45, M S_{\mathrm{e}}=0.05\right]$.

For the first TOT judgment, for the answerable questions, the participants experienced an average of $47 \%$ of unrecalled targets. For the unanswerable questions, the TOT rate was lower at $18 \%$. The participants were statistically more likely to experience a TOT for the answerable questions than for the unanswerable questions $[F(1,58)=$ 
$\left.207, M S_{\mathrm{e}}=0.01\right]$. This difference was also observed at the second judgment $\left[F(1,58)=134, M S_{\mathrm{e}}=0.02\right]$. We did an analysis comparing TOT rates for unanswerable questions with TOT rates for negative TOTs. There were significantly more negative TOTs than illusory TOTs $\left[F(1,50)=31.81, M S_{\mathrm{e}}=0.01\right]$.

Accuracy of tip-of-the-tongue states for answerable question. Resolution was more likely to occur after a commission error (.17) than after an omission error [.04; $\left.F(1,35)=19.38, M S_{\mathrm{e}}=0.04\right]$. There was also a main effect of type of TOT and the likelihood of resolution $\left[F(2,79)=5.73, M S_{\mathrm{e}}=0.01 ;\right.$ see Table 2B]. Post hoc tests showed that resolution was more likely to occur after an emotional TOT (.14) than after a nonemotional TOT $(.09)$ or an n-TOT (.09). Note that this pattern differs from the pattern observed in Experiment 1. In Experiment 1, strong TOTs were more likely to be followed by resolution only after commission errors.

Overall, false resolution rates were low. However, false resolutions were more likely to occur after initial commission errors $(.12)$ than after omission errors $[.06 ; F(1,50)=$ $\left.74.53, M S_{\mathrm{e}}=0.004\right]$. False resolutions were not more likely to occur after emotional TOTs (.09) than after nonemotional TOTs $\left[.07 ; F(2,100)=1.98, M S_{\mathrm{e}}=0.14\right]$.

The final recognition test was used as a measure of accuracy. Using the first TOT as the criterion TOT, recognition was higher after an emotional TOT than after a nonemotional TOT, which was higher than after an nTOT $\left[F(2,116)=58.88, M S_{\mathrm{e}}=0.02\right]$. Using the second TOT as the criterion TOT, recognition was again higher after an emotional TOT than after a nonemotional TOT, which was higher than after an n-TOT $[F(2,104)=65.17$, $M S_{\mathrm{e}}=.03$; see Table 3B). The first TOT judgments showed a .59 gamma correlation with recognition; the second TOT judgments showed a .66 correlation with recognition. The difference in accuracy between the first TOT and the second TOT was not significant $(F<1)$, and both gamma correlations were significantly higher than chance.

Feeling-of-knowing judgments. The mean feelingof-knowing magnitude was higher for the answerable questions (42) than for the unanswerable questions [21; $\left.F(1,58)=164, M S_{\mathrm{e}}=78.6\right]$. Feeling-of-knowing judgments were also significant predictors of final recognition. The gamma correlation between feeling-of-knowing judgments and recognition was .42 , significantly different from zero. Feeling-of-knowing judgments were significantly correlated with the likelihood of a TOT. The gamma correlation between the first TOT judgment and the feeling of knowing was .48 , and the correlation between the second TOT judgment and the feeling of knowing was .36 .

\section{EXPERIMENT 3}

In Experiment 3, following Smith (1994), we asked the participants to distinguish between those TOTs that were accompanied by a sense of imminent retrieval and those that were not. James wrote that TOTs are charac- terized by "making us at moments tingle with the sense of our closeness" (James, 1890, p. 251). R. Brown and McNeill (1966) speculated that "near" TOTs (those TOTs followed by immediate resolution) might be qualitatively different than "far" TOTs (those TOTs not followed by immediate resolution). Smith (1994) speculated that imminence might be the feature of TOTs that distinguishes them from simple feelings of knowing. One unique prediction for Experiment 3 is that, if the TOT always includes a feeling of imminence, we should observe very few nonimminent TOTs. Second, assuming nonimminent TOTs do occur, they should be less likely to be followed by resolution of the target.

\section{Method}

Participants. The participants were 54 Florida International University students, who received partial course credit for their participation. Each participant was tested individually on a Macintosh computer during a session that lasted approximately $1 \mathrm{~h}$. Three participants did not complete the study and were not included in the analyses.

Materials. The stimuli for the experiment were identical to those used in Experiment 1. One answerable question was replaced, because it was almost always misspelled in Experiments I and 2. It was replaced with another question from the Nelson and Narens (1980) norms.

Procedure. The procedures were identical to those used in Experiment 1, with the following exceptions. The instructions for what constituted an imminent and a nonimminent TOT were as follows:

If you do not answer the question correctly or leave the answer blank, you will be asked whether or not you are in a tip-of-the-tongue state for the target answer. A tip-of-the-tongue state (abbreviated TOT) means that you feel as if it is possible that you could recall the target answer, and that you feel as if its recall is imminent. Sometimes, you may feel as if you are about to recall the target, and will do so if given enough time-imminence. Other times, you may feel the TOT state, think that you know the answer, but may not feel the target is immediately imminent. If you are in a TOT state that is IMMINENT indicate this kind of TOT by pressing the I key. If you are in a TOT state, but do not feel as if recall is imminent press the $T$ key. If you cannot recall the answer and are not in a TOT state, press the $\mathrm{N}$ key.

This was explained first by the experimenter and then was repeated on the computer just before the experiment began. The experimenter then started the computer program that ran the experiment.

\section{Results}

Recall and recognition. Correct recall at the first test was $38 \%$, close to the mean obtained in Experiments 1 and 2 . Overall, recognition of initially unrecalled items was $38 \%$.

Response times differed as a function of whether the initial response was correct, a commission error, or an omission error $\left[F(2,80)=16.32, M S_{\mathrm{e}}=5.04\right]$. Post hoc tests indicated that this effect was due to long response times for commission errors (12.2), relative to correct responses $(9.8 \mathrm{sec})$ and omission error responses $(9.8 \mathrm{sec})$. The mean number of commission errors was $26.6 \mathrm{per}$ participant, whereas the mean number of omission errors was 23.2 per participant.

Distribution of tip-of-the-tongue states across categories. The participants had no difficulties making the distinction between imminent and nonimminent TOTs. 
For the TOT judgment at Time 1, the participants used the imminent category at the same rate as the nonimminent category $(F<1)$. This disconfirmed our hypothesis that rememberers would view all TOTs as imminent. Indeed, for unanswerable (illusory) questions, nonimminent TOTs were more frequently reported than imminent TOTs $\left[F(1,50)=7.32, M S_{\mathrm{e}}=0.02\right.$; see Table 1C]. For the answerable questions, TOTs were more common after commission errors than after omission errors at the first judgment $\left[F(1,49)=140, M S_{\mathrm{e}}=0.01\right]$, and at the second judgment $\left[F(1,49)=20.06, M S_{\mathrm{e}}=0.02\right]$.

For the first TOT judgment, for the answerable questions, the participants experienced TOTs for an average of $51 \%$ of unrecalled targets. For the unanswerable questions, the TOT rate was lower at $19 \%$ (see Table 1C). An ANOVA showed that the participants were statistically more likely to experience TOTs for the answerable questions than for the unanswerable questions $[F(1,50)=$ $\left.296, M S_{\mathrm{e}}=0.01\right]$. This difference was also observed at the second judgment $\left[F(1,50)=142, M S_{\mathrm{e}}=0.02\right]$. We did an analysis comparing TOT rates for unanswerable questions with TOT rates for negative TOTs. There were significantly more negative TOTs than illusory TOTs $\left[F(1,48)=39.68, M S_{\mathrm{e}}=0.01\right]$.

Accuracy of tip-of-the-tongue states for answerable questions. Resolution was more likely to occur after a commission error (.16) than after an omission error [.03; $\left.F(1,49)=84.64, M S_{\mathrm{e}}=0.01\right]$. There was also a main effect of type of TOT and the likelihood of resolution $\left[F(2,78)=14.64, M S_{\mathrm{e}}=0.03\right.$; see Table $\left.2 \mathrm{C}\right]$. Post hoc tests showed that resolution was more likely to occur after an imminent TOT (.21) than after a nonimminent TOT (.08) or an n-TOT (.09).

False resolutions were more likely to occur after initial commission errors (.15) than after omission errors [.11; $\left.F(1,49)=18.35, M S_{e}=0.007\right]$. False resolutions were not more likely to occur after imminent TOTs $(.13)$ than after nonemotional TOTs $\left[.11 ; F(2,98)=1.70, M S_{\mathrm{e}}=0.03\right]$.

We also examined the relation between TOTs and recognition. Using the first TOT as the criterion TOT, recognition was higher after a TOT than after an n-TOT $\left[F(2,80)=78.21, M S_{\mathrm{e}}=0.03\right]$. Imminent TOTs were not more likely to be recognized than nonimminent TOTs (see Table $3 \mathrm{C}$ ). There was a significant interaction between type of TOT judgments and the time at which the judgment was made $\left[F(2,80)=11.42, M S_{\mathrm{c}}=0.02\right]$. Using the second TOT as the criterion TOT, recognition was higher for imminent TOTs than for nonimminent TOTs, which was higher than that for n-TOTs (see Table 3C). The first TOT judgments showed a .55 gamma correlation with recognition; the second TOT judgments showed a .65 correlation with recognition. The gamma correlation between the second TOT judgments and recognition was significantly higher than the gamma correlation between the first TOT judgments and recognition $\left[F(1,50)=4.21, M S_{\mathrm{e}}=0.07\right]$. Both gamma correlations were significantly higher than chance.
Feeling-of-knowing judgments. The mean feelingof-knowing magnitude was higher for the answerable questions (45) than for the unanswerable questions [24; $\left.F(1,50)=148, M S_{\mathrm{e}}=81\right]$. Feeling-of-knowing judgments were also significant predictors of final recognition. The gamma correlation between feeling-of-knowing judgments and recognition was .42 , significantly different from zero. Feeling-of-knowing judgments were significantly correlated with the likelihood of a TOT, but only for the first TOT judgment. The gamma correlation between the first TOT judgment and feeling of knowing was .33 (significantly higher than zero), and the correlation between the second TOT judgment and feeling of knowing was .09 (not significantly higher than zero). Because imminent TOTs were ranked higher than nonimminent TOTs when the gammas were calculated, the low correlation observed here reflects the fact that imminence and feeling of knowing are not correlated, not that TOTs and feeling of knowing are not correlated.

\section{GENERAL DISCUSSION}

We begin the discussion with a review of the main results. Three experiments examined phenomenological aspects of real and illusory TOTs. The phenomenological aspects included the strength, emotionality, and imminence of TOTs. Across all three experiments, the participants were able to differentiate between TOT substates. These substates were correlated with objective measures of memory, although the pattern differed, depending on which substates were being used. Imminence was a better predictor of resolution than was strength or emotionality. Imminence was not a good predictor of recognition, at least at the first TOT judgment. Strength, although not emotionality or imminence, predicted false resolution. Furthermore, the participants were less likely to assign TOTs to unanswerable questions, and the TOT given to unanswerable questions tended to be weaker, less emotional, and less imminent than those given to answerable questions.

These experiments show that participants can divide their TOTs into substates and can do so in a way that is predictive of memory performance. The findings suggest that TOTs are not simply dichotomous states, as they are usually taken to be in the literature (see A. S. Brown, 1991; Schwartz, 1999). TOTs appear to be multidimensional subjective states. TOTs have been divided into substates as a function of the objective characteristics of target memory in past research (Burke et al., 1991; Jones \& Langford, 1987; Koriat \& Lieblich, 1974; Vigliocco et al., 1997). In the present study, TOTs were divided into substates on the basis of the characteristics of the phenomenological experiences (i.e., strength, emotionality, and imminence) rather than on the basis of the memory outcome. Future research could be directed at other aspects of the phenomenology of TOTs, such as the "aha" that occurs when a TOT is resolved or the relief that may occur after a TOT has been resolved. 
TOT phenomenology, although mostly neglected, is an important topic to explore, because recent research has demonstrated that TOTs are not always associated with target knowledge (e.g., Schwartz, 1998; Schwartz \& Smith, 1997; Widner et al., 1996). Therefore, it is not correct to always associate TOTs with target retrieval, and it is fruitful to look at the processes that cause TOT phenomenology, as well as at the retrieval processes during a TOT (Schwartz, 1999). The present set of experiments is an initial effort to explore TOT phenomenology.

\section{Illusory Tip-of-the-Tongue States}

Following Schwartz (1998), we included a set of questions that did not have correct answers. Schwartz found that some unanswerable questions elicited TOTs, despite the impossibility of target knowledge. The TOTs to these unanswerable questions were labeled as illusory TOTs. In the present study, it was hypothesized that illusory TOTs would show, on average, a different profile on the subjective measures assessed here. More specifically, we predicted that illusory TOTs would be weaker, less emotional, and less imminent than TOTs for answerable questions. This hypothesis was confirmed in the present experiments (see Table 1).

One criticism of the use of illusory TOTs in these experiments is that they may be caused by failures of source monitoring (Johnson, Hashtroudi, \& Lindsay, 1993) rather than of true TOT feelings. The source monitoring account suggests that illusory TOTs may be akin to the Moses illusion (Kamas, Reder, \& Ayers, 1996). ${ }^{1}$ In the Moses illusion, participants fail to recognize that an error exists in the question (e.g., How many animals did Moses take on his ark?). Thus, they will often make a response without realizing the error. Similarly, illusory TOTs may be better thought of as TOTs, but for an item that is not the correct answer. The error that participants make lies in not recognizing the question as false. However, this view suggests that the TOTs for the false question should not be different from other TOTs in its phenomenological characteristics. Nonetheless, the illusory TOTs were weaker, less emotional, and less imminent than other TOTs, including negative TOTs (TOTs followed by incorrect recognition). A second explanation of illusory TOTs suggests that they might result from demand characteristics in the experiment (Widner et al., 1996). However, illusory TOTs that were initially strong, emotional, or imminent tended to remain strong, emotional, and imminent from the first judgment to the second, suggesting that the strong TOT feeling was not an arbitrary judgment, but something that reflected the rememberer's subjective state.

\section{Response Times for Correct Items and Commission Errors}

Across all three experiments, we found that correct answers were given more quickly than commission er- rors in the recall phase. The response times to make omission errors was variable across experiments but was consistently less than the response times to the commission errors. There are two possible explanations for these data. First, the longer response times may indicate that participants are less confident in their commission errors and that the lower confidence is reflected in their slow responses. Second, longer response times may represent a general feature of incorrect recall or false memories. Given the recent surge of interest in false memories, the issue of differential response times may be illuminating (see Roediger, 1996).

\section{Conclusions}

The present study represents an initial attempt to examine phenomenological aspects of the TOT. To date, most research has examined the relation between TOTs and retrieval and the etiology of TOTs (see A. S. Brown, 1991). In these experiments, we found that rememberers classified their TOTs according to strength, emotional content, and imminence and that these substates correlated with objective performance. Moreover, illusory TOTs tended to be weaker, less emotional, and less imminent than real TOTs. Recently, metacognition has been making progress at the scientific investigation of human consciousness and phenomenology (e.g., Nelson, 1996). We hope that the present experiments will contribute to the growing interest in phenomenology.

\section{REFERENCES}

Brennan, T., Baguley, T., Bright, J., \& Bruce, V. (1990). Resolving semantically induced tip-of-the-tongue states for proper nouns. $\mathrm{Mem}$ ory \& Cognition, 18, 339-347.

Brown, A. S. (1991). A review of the tip-of-the-tongue experience. Psychological Bulletin, 109, 204-223.

Brown, R., \& MCNEILl, D. (1966). The "tip of the tongue" phenomenon. Journal of Verbal Learning \& Behavior, 5, 325-337.

Burke, D. M., Mackay, D. G., Worthley, J. S., \& Wade, E. (1991). On the tip of the tongue: What causes word finding failures in young and older adults? Journal of Memory \& Language, 30, 542-579.

Gardiner, J. M., Craik, F. I. M., \& Bleasdale, F. A. (1973). Retrieval difficulty and subsequent recall. Memory \& Cognition, 1, 213-216.

Harley, T. A., \& Bown, H. E. (1998). What causes a tip-of-the-tongue state? Evidence for lexical neighbourhood effects in speech production. British Journal of Psychology, 89, 151-174.

JAMES, W. (1890). The principles of Psychology. New York: Holt.

Johnson, M. K., Hashtroudi, S., \& Lindsay, D. S. (1993). Source monitoring. Psychological Bulletin, 114, 3-28.

Jones, G. V., \& LangFord, S. (1987). Phonological blocking in the tip of the tongue state. Cognition, 26, 115-122.

Kamas, E. N., Reder, L. M., \& Ayers, M. S. (1996). Partial matching in the Moses illusion: Response bias not sensitivity. Memory \& Cognition, 24, 687-699.

Kohn, S. E., Wingfield, A., Menn, L., Goodglass, H., Berko GleaSON, J., \& HYDE, M. (1987). Lexical retrieval: The tip-of-the-tongue phenomenon. Applied Psycholinguistics, 8, 245-266.

KORIAT, A. (1993). How do we know that we know? The accessibility account of the feeling of knowing. Psychological Review, 100, 609-639.

Koriat, A., \& LIEBLICH, I. (1974). What does a person in a "TOT" state know that a person in a "don't know" state doesn't know? Memory \& Cognition, 2, 647-655. 
KozLowsKI, L. (1977). Effects of distorted auditory and of rhyming cues on retrieval of tip-of-the-tongue words by poets and nonpoets. Memory \& Cognition, 5, 477-481.

Krinsky, R., \& Nelson, T. O. (1985). The feeling of knowing for different types of retrieval failure. Acta Psychologica, 58, 141-158.

Metcalfe, J., Schwartz, B. L., \& Joaquim, S. G. (1993). The cue familiarity heuristic in metacognition. Journal of Experimental Psychology: Learning, Memory, \& Cognition, 19, 851-861.

Miozzo, M., \& Caramazza, A. (1997). Retrieval of lexical-syntactic features in tip-of-the-tongue states. Journal of Experimental Psychology: Learning, Memory, \& Cognition, 23, 1410-1423.

NELSON, T. O. (1984). A comparison of current measures of the accuracy of feeling-of-knowing predictions. Psychological Bulletin, 95, 109-133.

Nelson, T. O. (1988). Predictive accuracy of feeling of knowing across different criterion tasks and across different subject populations and individuals. In M. Gruneberg, P. Morris, \& R. Sykes (Eds.), Practical aspects of memory: Current research and issues (Vol. 1, pp. 190196). New York: Wiley.

Nelson, T. O. (1996). Consciousness and metacognition. American Psychologist, 51, 102-116.

NeLSON, T. O., \& NARENS, L. (1980). Norms of 300 general-information questions: Accuracy of recall, latency of recall, and feeling-of-knowing ratings. Journal of Verbal Learning \& Verbal Behavior, 19, 338-368.

Perfect, T. J.. \& Hanley, J. R. (1992). The tip-of-the-tongue phenomenon: Do experimenter-presented interlopers have any effect? Cognition, 45, 55-75.

REASON, J. T., \& LuCAS, D. (1984). Using cognitive diaries to investigate naturally occurring memory blocks. In J. Harris \& P. E. Morris (Eds.), Everyday memory, actions, and absent mindedness (pp. 53-70). London: Academic Press.

RoEDigeR, H. L., III (1996). Memory illusions. Journal of Memory \& Language, 35, 76-100.

SChWARTZ, B. L. (1998). Illusory tip-of-the-tongue states. Memory, 6 , 623-642.
SchwarTZ, B. L. (1999). Sparkling at the end of the tongue: The etiology of tip-of-the-tongue phenomenology. Psychonomic Bulletin \& Review, 6, 379-393.

SCHWARTZ, B. L., \& SMITH, S. M. (1997). The retrieval of related information influences tip-of-the-tongue states. Journal of Memory \& Language, 36, 68-86.

SмIтH, S. M. (1994). Frustrated feelings of imminent recall: On the tipof-the-tongue. In J. Metcalfe \& A. P. Shimamura (Eds.), Metacognition: Knowing about knowing (pp. 27-46). Cambridge: MIT Press.

Tulving, E. (1989). Memory: Performance, knowledge, and experience. European Journal of Cognitive Psychology, 1, 3-26.

Vigliocco, G., Antonini, T., \& Garrett, M. F. (1997). Grammatical gender is on the tip of Italian tongues. Psychological Science, $\mathbf{8}$, 314-317.

Widner, R. L., Smith, S. M., \& Graziano, W. G. (1996). The effects of demand characteristics on the reporting of tip-of-the-tongue states and feeling-of-knowing states. American Journal of Psychology, 109, 525-538.

WILKINSON, T. S., \& NELSON, T. O. (1984). FACTRETRIEVAL2: A PASCAL program for assessing someone's recall of generalinformation facts, confidence about recall correctness, feeling-ofknowing judgments for nonrecalled facts, and recognition of nonrecalled facts. Behavior Research Methods, Instruments, \& Computers, 16, $486-488$

\section{NOTE}

1. We are grateful to an anonymous reviewer for suggesting the relation between illusory TOTs and the Moses illusion.

(Manuscript received April 15, 1998; revision accepted for publication January 13, 1999.) 